\title{
Singlet Fission in Concentrated TIPS-Pentacene Solutions: The Role of Excimers and Aggregates
}

Miroslav Dvořák, Shyamal Prasad, Cameron Dover, Chelsea Forest, Akasha Kaleem, Rowan W. MacQueen, Anthony J Petty, Roslyn Forecast, John E. Anthony, Murad J. Y. Tayebjee, Asaph N. Widmer-Cooper, Pall Thordarson, Timothy Schmidt

Submitted date: 03/06/2021 - Posted date: 04/06/2021

Licence: CC BY-NC-ND 4.0

Citation information: Dvořák, Miroslav; Prasad, Shyamal; Dover, Cameron; Forest, Chelsea; Kaleem, Akasha; MacQueen, Rowan W.; et al. (2021): Singlet Fission in Concentrated TIPS-Pentacene Solutions: The Role of Excimers and Aggregates. ChemRxiv. Preprint. https://doi.org/10.26434/chemrxiv.14727708.v1

The exciton dynamics of 6,13-bis(triisopropylsilyl-ethynyl) pentacene is investigated to determine the role of excimer and aggregate formation in singlet fission in high concentration solutions.

Photoluminescence spectra were measured by excitation with the evanescent wave in total internal reflection, in order to avoid reabsorption effects. The spectra over nearly two magnitudes of concentration were near identical, with no evidence for excimer emission. Time-correlated single-photon counting measurements confirm that the fluorescence lifetime shortens with concentration, and we obtain a bimolecular rate constant of $\$ 4 \mid$ times $10^{\wedge} 9 \$ \backslash, M \$^{\wedge}\{-1\} \$ s \$^{\wedge}\{-1\} \$$ in chloroform. The observed rate constant grows at high concentrations. This effect is modelled in terms of the hard sphere radial distribution function.

NMR measurements confirm that aggregation takes place with a binding constant of between 0.14 and $0.43 \mathrm{M} \$ \wedge\{-1\} \$$. Transient absorption measurements are consistent with a diffusive encounter mechanism for singlet fission, with hints of more rapid singlet fission in aggregates at the highest concentration measured. These data show that excimers do not play the role of an emissive intermediate in exothermic singlet fission in solution, and that while aggregation occurs at higher concentrations, the mechanism of singlet fission remains dominated by diffusive encounters.

File list (2)

main.pdf (5.49 MiB)

view on ChemRxiv - download file

SI.pdf (4.15 MiB)

view on ChemRxiv - download file 


\section{Singlet fission in concentrated TIPS-pentacene solutions: The role of excimers and aggregates}

Miroslav Dvořák, ${ }^{\dagger} \ddagger$ Shyamal K. K. Prasad, ${ }^{\dagger}$ Cameron B. Dover, ${ }^{\dagger}$ Chelsea R. Forest, ${ }^{\circledR}$ Akasha Kaleem, ${ }^{\S}$ Rowan W. MacQueen, ${ }^{\prime}$ Anthony J. Petty II, ${ }^{\perp}$ Roslyn Forecast,\# John E. Anthony, ${ }^{\perp}$ Murad J. Y. Tayebjee, ${ }^{\S}$ Asaph Widmer-Cooper, ${ }^{\circledR}$ Pall Thordarson, ${ }^{\circledR}$ and Timothy W. Schmidt*,

$\dagger$ †ARC Centre of Excellence in Exciton Science, School of Chemistry, UNSW Sydney, NSW 2052, Australia

$\ddagger$ Department of Physical Electronics, Faculty of Nuclear Sciences and Physical Engineering, Czech Technical University in Prague, V Holešovičkách 2, 18000 Prague 8, Czechia

TAustralian Centre for Nanomedicine and The ARC Centre of Excellence in Convergent Bio-Nano Science and Technology, School of Chemistry, UNSW Sydney, NSW 2052, Australia

$\S$ School of Photovoltaic and Renewable Energy Engineering, UNSW Sydney, NSW 2052, Australia

||Department of Spins in Energy Conversion and Quantum Information Science, Helmholtz-Zentrum Berlin für Materialen und Energie GmbH, Berlin, Germany

$\perp$ Department of Chemistry, University of Kentucky, Lexington, Kentucky 40506, United States

\#ARC Centre of Excellence in Exciton Science, School of Science, RMIT University, Melbourne, VIC, 3001,

Australia

@ARC Centre of Excellence in Exciton Science, School of Chemistry, The University of Sydney, NSW 2006, Australia

E-mail: timothy.schmidt@unsw.edu.au 


\begin{abstract}
The exciton dynamics of 6,13-bis(triisopropylsilyl-ethynyl) pentacene is investigated to determine the role of excimer and aggregate formation in singlet fission in high concentration solutions.

Photoluminescence spectra were measured by excitation with the evanescent wave in total internal reflection, in order to avoid reabsorption effects. The spectra over nearly two magnitudes of concentration were near identical, with no evidence for excimer emission. Timecorrelated single-photon counting measurements confirm that the fluorescence lifetime shortens with concentration, and we obtain a bimolecular rate constant of $4 \times 10^{9} \mathrm{M}^{-1} \mathrm{~s}^{-1}$ in chloroform. The observed rate constant grows at high concentrations. This effect is modelled in terms of the hard sphere radial distribution function.

NMR measurements confirm that aggregation takes place with a binding constant of between 0.14 and $0.43 \mathrm{M}^{-1}$. Transient absorption measurements are consistent with a diffusive encounter mechanism for singlet fission, with hints of more rapid singlet fission in aggregates at the highest concentration measured.

These data show that excimers do not play the role of an emissive intermediate in exothermic singlet fission in solution, and that while aggregation occurs at higher concentrations, the mechanism of singlet fission remains dominated by diffusive encounters.
\end{abstract}

\title{
Introduction
}

Singlet fission is a bichromophoric process in which the energy of an excited singlet state apportions into independent triplet states on separate chromophores. ${ }^{1,2}$ It is presently a subject of intense interest, due to its applicability to excitonic solar cells with the potential to circumvent the singlethreshold detailed-balance efficiency limit by creating more than one excited electron-hole pair for each incoming high-energy photon. ${ }^{3-6}$

While endothermic singlet fission $\left(E\left(2 T_{1}\right)>E\left(S_{1}\right)\right)$ is required to reach calculated efficiency limits, ${ }^{7}$ it is in practice less efficient than exothermic singlet fission, and must compete with trap- 
ping processes such as excimer formation. ${ }^{8,9}$ Pentacene chromophores are archetypal exothermic singlet fission materials which are intensively studied as they exhibit high-efficiency singlet fission approaching $200 \%$ quantum yield. ${ }^{10-13}$

The highly soluble derivative, 6,13-bis(triisopropylsilylethynyl) pentacene (TPn) was shown by Walker et al. to exhibit singlet fission in highly concentrated solutions $(\leq 0.075 \mathrm{M}) .{ }^{14}$ In that work it was argued, on the basis of the appearance of steady-state spectra, that emissive excimer formation occurred as part of the singlet fission process. This is an important point given the present controversy concerning the role of excimers in singlet fission, and the extensive effort in uncovering the detailed mechanism. ${ }^{9,14-19}$ However, performing steady-state spectroscopy with highly concentrated solutions is difficult, due to severe reabsorption effects which can hinder the interpretation of observed spectra. The study of Walker et al. specifically ruled out any molecular aggregation. Yet, a recent report by Grieco and co-workers concludes that TPn molecules aggregate through their TIPS side-groups, and that singlet fission does not occur by diffusive encounters of independent chromophores. ${ }^{20}$ This apparent contradiction warrants further investigation.

In the present work, we revisit the steady-state spectroscopy of TPn at high concentrations using an excitation set-up which exploits the shallow penetration-depth of the evanescent wave generated under total-internal reflection conditions. We show that with such an experimental geometry, there is no evidence for excimer formation, contrary to the report of Walker et al. ${ }^{14} \mathrm{We}$ show by NMR spectroscopy that aggregation does occur, but demonstrate using transient absorption spectroscopy that singlet fission principally occurs due to encounter-complex formation on the time-scale of the radiative decay. The rate of singlet fission was found to be non-linear in concentration, which we ascribe to molecular crowding effects. 

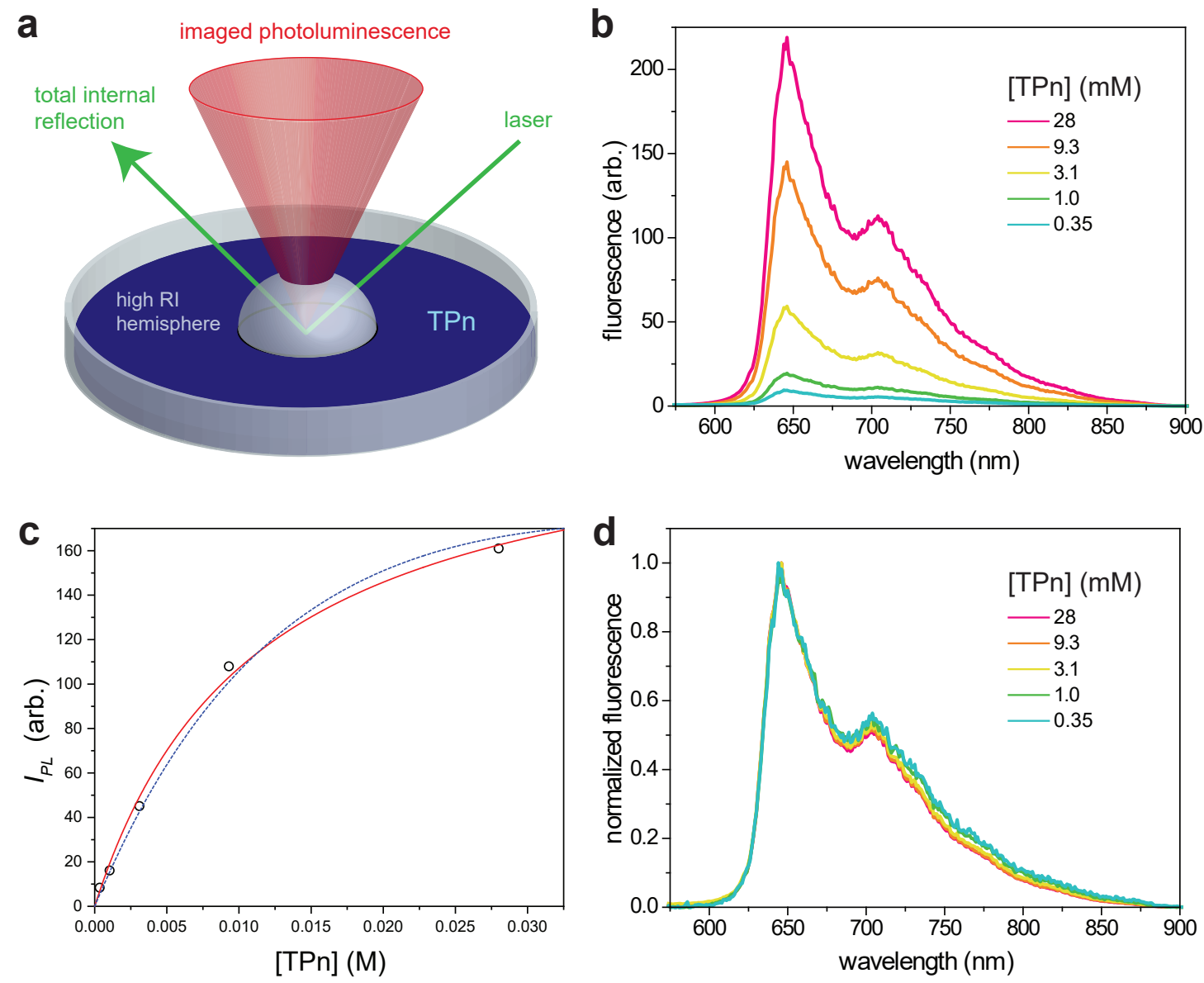

Figure 1: a. A $532 \mathrm{~nm} \mathrm{cw}$ laser is directed to the centre of the flat face of a high refractive index hemisphere which is immersed in TIPS-pentacene, and not in contact with the vessel. The beam undergoes total internal reflection, with the evanescent wave exciting only molecules within about $100 \mathrm{~nm}$ of the interface. The small volume of photoluminescence is collected and analysed by a spectrograph. b. Steady-state photoluminescence spectra of TPn as a function of concentration. c. Observed photoluminescence intensity (arbitrary units) as a function of [TPn]. The deviation from linearity is due to concentration quenching caused by singlet fission. The solid line is the model given by Equations 1 and 2, with $1 /\left(k_{r}+k_{n r}\right)=12$ ns (vide infra, $c f$. Ref. 14) and $k_{q}=(7.5 \pm 1.0) \times 10^{9} \mathrm{M}^{-1} \mathrm{~s}^{-1}$. The dashed curve is a fit using the quenching behaviour from the TCSPC experiments. d. Normalized steady-state photoluminescence spectra of TPn as a function of concentration. 


\section{Experimental}

\section{Steady-State Spectroscopy}

The experimental set-up is shown in Figure 1a. A $532 \mathrm{~nm}$ continuous laser source was directed into a hemisphere of high-refractive index material (S-LAH79, $n=2$, Edmund Optics) immersed in TPn solution in chloroform, at an angle of $66.5^{\circ}$ to the normal of the S-LAH79/chloroform $(n=1.44)$ interface. The optic was supported by a teflon ring, such that its central region was not in contact with anything but the solution of interest. The beam was totally internally reflected and the evanescent wave excited a shallow depth of TPn solution in chloroform. The depth of the excitation volume can be calculated from the laser beam impact angle and refractive indices of chloroform and the glass hemisphere, respectively. The penetration of the excitation intensity is $40 \mathrm{~nm}(1 / e)$. The photoluminescence was collected above the hemisphere and analysed with a spectrograph (Princeton).

\section{Time-Resolved Spectroscopy}

Time-correlated single-photon counting experiments were performed in reflection geometry with a Horiba-Jobin Fluorolog spectrometer and a IBH NanoLED-560 excitation source, housed in the Prague laboratory.

We performed transient absorption spectroscopy in two laboratories respectively housed in the School of Photovoltaic and Renewable Energy Engineering (SPREE) and the School of Chemistry (SoC). In the Spectroscopy for Renewable Energy (SpectRE) laboratory in SPREE, we investigated the excited-state dynamics of TPn using a commercial spectrometer (Ultrafast systems, Helios). The system provides $\approx 8 \mathrm{~ns}$ optical delay line with a resolution of $\approx 14 \mathrm{fs}$. The amplified laser system generates $800 \mathrm{~nm}$ pulses at a repetition rate of $1 \mathrm{kHz}$. The $800 \mathrm{~nm}$ pulse was focused onto a Ti-Sapphire oscillator to generate white light continuum probe, detected with a 256-pixel linear InGaAs diode array camera for the near-IR region $(800-1600 \mathrm{~nm})$. Second harmonic pulses of the pump beam $(400 \mathrm{~nm}$, fwhm $100 \mathrm{fs}, 500 \mathrm{~Hz})$ were generated to excite the sample. At least 
three individual scans were taken, and each scan was averaged over 1 second per time delay. The polarization of the pump pulse was scrambled.

In the SoC laboratory, we performed transient absorption spectroscopy using a home-built set-up, as detailed elsewhere. ${ }^{21}$ Briefly, the broadband probe pulse was produced by focusing a femtosecond laser pulse (785 nm, 1000 Hz, Clark-MXR CPA 2210, 150 fs, Ti:sapph. regen. amp.) onto an yttrium aluminium garnet crystal. Two pump sources were used, depending on the timescale of interest. For time-scales up to a few ns, the pump was generated by a 2-stage optical parametric amplifier (TOPAS-C, light conversion), delayed by a motorized stage (Thorlabs, LTS300). For longer time-scales $<\mathrm{ms}$, the electronically delayed $532 \mathrm{~nm}$ output of a $\mathrm{Nd}$ :YAG laser was used (InnoLas picolo, 800 ps pulse-width). The probe pulse was polarized perpendicularly to the pump pulse.

\section{NMR Spectroscopy}

Nuclear Magnetic Resonance (NMR) spectra were recorded on a Bruker Avance III (400 MHz) spectrometer; all spectra were recorded at $298 \mathrm{~K}$. Samples were dissolved in $\mathrm{CDCl}_{3}$ to a known concentration with a final volume of $600 \mu \mathrm{L}$, (residual solvent peak for $\mathrm{CDCl}_{3}$ : 7.26 ppm). All NMR data were acquired using Bruker Topspin 3.5 Software.

\section{Results and Discussion}

\section{Steady-State Spectroscopy}

In Figure $1 \mathrm{~b}$ we plot the observed photoluminescence (PL) spectrum at five different concentrations, spanning 0.35 to $28 \mathrm{mM}$. The intensity of the PL increases with concentration, as expected, as there are more molecules in the excitation volume, which is largely determined by the exponent of the evanescent wave (Figure 1c). The peak extinction coefficient is about $27000 \mathrm{M}^{-1} \mathrm{~cm}^{-1}$, and thus at the highest concentration, a non-evanescent beam would excite a depth of approximately 
$13 \mu \mathrm{m}(A=1)$. By contrast, the evanescent wave penetrates only $100 \mathrm{~nm}$, and thus on this scale all solutions can be considered optically thin.

The spectra, normalized to the $0-0$ band, are shown in Figure 1d. There is hardly a difference between the spectra, and if anything, the highest concentrations exhibit the least intense $0-1$ bands, contrary to expectation if excimers were to contribute in a significant way to the photoluminescence. Furthermore, the identical shape of the $0-0$ bands underlines the lack of reabsorption effects in our experimental geometry. Our results accord with those of Schaberle et al., who report essentially unchanged emission spectra for TPn in toluene up to a concentration of $50 \mathrm{mM} .^{22}$ The lack of concentration-dependent emission spectra at longer wavelengths also accords with the results of Walker et al. ${ }^{14}$ and Grieco et al. ${ }^{20}$ Absorption spectra are also unchanged up to $30 \mathrm{mM}$ (see SI), also demonstrated by Schaberle et al. in toluene. ${ }^{22}$

Immediate evidence of singlet fission in this experiment comes from the concentration-dependent photoluminescence quantum yield $\left(\Phi_{P L}\right)$. If the rate of dynamic quenching is given by $k_{q}[T P n]$, then the $\Phi_{P L}$ will be given by

$$
\Phi_{P L}=\frac{k_{r}}{k_{r}+k_{n r}+k_{q}[T P n]}
$$

where $k_{r}$ is the radiative decay rate at infinite dilution, and $k_{n r}$ is the corresponding rate of nonradiative decay.

The observed PL intensity will scale with the concentation of absorbers and their quantum yield,

$$
I_{P L} \propto[T P n] \Phi_{P L}
$$

In Figure 1c, we plot the observed photoluminescence intensity as a function of concentration. The deviation from linearity is a sign of concentration quenching, and in this case is understood to be due to singlet fission. ${ }^{14}$ The solid line is a fit to a single second-order rate constant. The quenching constant is $k_{q}=(7.5 \pm 1.0) \times 10^{9} \mathrm{M}^{-1} \mathrm{~s}^{-1}$, which is higher than reported by Walker et al. ${ }^{14}$, but still below the diffusion limit, which is about $1.2 \times 10^{10} \mathrm{M}^{-1} \mathrm{~s}^{-1}$ for chloroform at $294 \mathrm{~K}$. However, 
as will be seen below, things are not quite this simple.

A more direct measurement of $k_{q}$ comes from measurements of the photoluminescence lifetime as a function of concentration. The decay traces are plotted in Figure 2a. The traces were fit to both a single and double exponential decay convolved with the instrument response function (IRF). The double exponential fits principally improved the residuals within the IRF and the major decay rates were not substantially different. The fitted decay constants as a function of concentration are shown in Figure 2b.

It is clear that both single and double exponential fits show that the rate constant for selfquenching is non-linear in concentration. The upward curve in Figure $2 b$ deserves attention. Keizer explained that both upward and downward curving Stern-Volmer plots can be rationalized by the diffusive sampling of the radial distribution function of quencher around a chromophore. ${ }^{23}$ This amounts to saying that the structure of the solution impacts on the statistics of meeting a collision partner within the radiative lifetime. As the concentration increases, the part of the radial distribution function being sampled is less homogeneous, and positive deviations are typically seen for concentrations exceeding $10 \mathrm{mM} .^{23}$

We model the observed quenching rate constant, $k_{q}^{o b s}$, in terms of the Smoluchowski reactivity

$$
k_{q}^{o b s}=k_{q}^{0} g(R)
$$

where $R$ is the interaction distance, normally taken to be the molecular diameter for short-range reactions. ${ }^{24}$ For hard spheres, it has been shown that

$$
g(R)=\frac{\left(1+\frac{1}{2} \phi\right)}{(1-\phi)^{2}}
$$

where the volume fraction $\phi$,

$$
\phi=\frac{\pi \rho R^{3}}{6}=\frac{\pi N_{A}[T P n] R^{3}}{6}
$$

with $R$ in $\mathrm{dm}^{.25,26}$ 

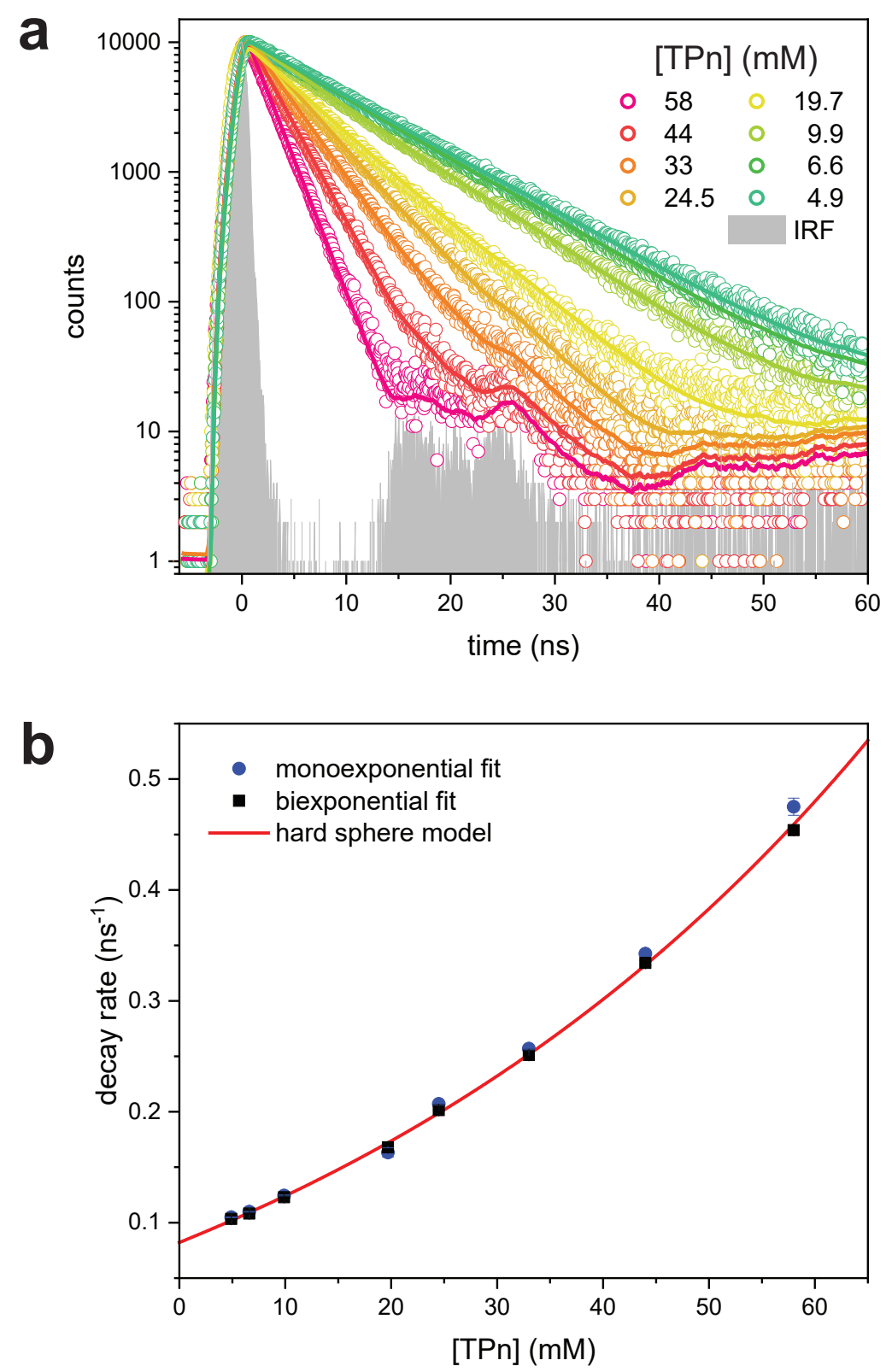

Figure 2: a. Measured TCSPC decay curves of TPn as a function of concentration. The grey, shaded curve is the instrument response function. $b$. The fitted decay rate constant as a function of concentration. 
Combining the above equations we obtain an expression for the observed lifetime, $\tau$,

$$
1 / \tau=k([T P n])=\left(k_{r}+k_{n r}\right)+k_{q}^{0} g(R,[T P n])[T P n]
$$

The fit to Equation 6 is shown in Figure 2b. The fit yields an intrinsic second order rate constant of $k_{q}^{0}=3.86(18) \times 10^{9} \mathrm{M}^{-1} \mathrm{~s}^{-1}$ and a dilute $S_{1}$ state decay rate of $8.22(14) \times 10^{7} \mathrm{~s}^{-1}$ $\left(k_{r}+k_{n r}, \equiv 12.17(21) \mathrm{ns}\right)$. The upward curve is controlled by a single parameter, $R$, the hard sphere interaction distance, which is fit here by $2.19(9) \mathrm{nm}$. A linear fit to the lowest three concentrations yields an intercept of $8.3(3) \times 10^{7} \mathrm{~s}^{-1}(12.0(4) \mathrm{ns})$ and a slope of $4.0(4) \times 10^{9} \mathrm{M}^{-1} \mathrm{~s}^{-1}$.

Walker et al. reported that the singlet fission probability reached $50 \%$ at $7.9 \mathrm{mM}$, with a radiative lifetime of $13 \mathrm{~ns}$. This would correspond to $k_{q}=9.7 \times 10^{9} \mathrm{M}^{-1} \mathrm{~s}^{-1}$, which is significantly higher than their reported bimolecular rate constant of $2.2 \times 10^{9} \mathrm{M}^{-1} \mathrm{~s}^{-1}$. Figure 5 of Reference 14 shows the fitted decay of a $75 \mathrm{mM}$ solution to a single exponential. The claimed time constant of 400 ps would imply a bimolecular rate constant of $3.2 \times 10^{10} \mathrm{M}^{-1} \mathrm{~s}^{-1}$, well in excess of the diffusion limit. But, the data shows that the emission decays to $1 \%$ over about $10 \mathrm{~ns}$, which implies a lifetime of about $2 \mathrm{~ns}$, which is consistent with the present findings.

Our obtained dilute $S_{1}$ state lifetime, $12.2 \mathrm{~ns}$, is slightly shorter than reported by Walker et al., consistent with a small oxygen quenching rate constant of $k_{\mathrm{O}_{2}}=1.2-1.8 \times 10^{7} \mathrm{M}^{-1} \mathrm{~s}^{-1} \cdot{ }^{27}$ Walker et al. reported differences in the lifetimes measured at different emission wavelengths as evidence of excimer formation. ${ }^{14} \mathrm{We}$ found no evidence for this in our experiments.

A fit to the photoluminescence intensity is shown in Figure 1c using the rate constants fit in Figure $2 \mathrm{~b}$ as a dashed line. Given the difficulty of maintaining a constant concentration in the experiment, due to solvent evaporation, the fit is acceptable.

Aggregates themselves are expected to be revealed by a shorter lifetime, resulting in biexponential radiative decay kinetics. Yet, within the time resolution of the TCSPC experiment (50 ps), this was not clearly in evidence. It was reported by Grieco et al. that, in chlorinated solvents, TPn molecules aggregate through association of their TIPS groups. ${ }^{20}$ Walker et al. ruled out aggrega- 
tion on the basis of diffusion NMR results, ${ }^{14}$ but as we shall show, our data strongly supports a small degree of aggregation.

\section{${ }^{1}$ H Nuclear Magnetic Resonance}

To investigate if TPn formed aggregates, a NMR dilution study was carried out. The results show up to $0.1 \mathrm{ppm}$ shift in the ${ }^{1} \mathrm{H}$ NMR spectra for some of the TPn proton resonances when the concentration was varied between $0.0018-0.25 \mathrm{M}$. This data was fitted to aggregation models described first by Martin $^{29}$ and then deployed by us (PT) on the supramolecular.org website for calculating binding constants. ${ }^{30}$ Two models were employed; the equal-K (EK) or isodesmic model ${ }^{29}$ and the cooperative EK (coEK) ${ }^{29,31}$ binding model. Fitting the EK and coEK models to the data indicates that the simpler EK (isodesmic) model is sufficient in terms of describing the data and using the more complex coEK is not justifiable using the Bayesian Information Criteria (BIC) ${ }^{32}$ as a robust method for model comparison. The dilution study between $0.0018-0.25 \mathrm{M}$ yielded an aggregation constant $K_{E}=0.29 \mathrm{M}^{-1}$ using the EK (isodesmic) aggregation model (Figure 3). Using a Monte Carlo approach ${ }^{28}$ we obtained a $95 \%$ confidence interval (C.I.) for $K_{E}=0.14-0.43 \mathrm{M}^{-1}$.

The proportion of free chromophores under the EK model is given by ${ }^{29,31}$

$$
\alpha=\frac{2 K_{E}[T P n]+1-\sqrt{4 K_{E}[T P n]+1}}{2 K_{E}^{2}[T P n]^{2}}
$$

At concentration of $0.050 \mathrm{M}$ this indicates that about $1.6 \%$ (1.4-4.2\% using the $95 \%$ C.I.) of the TPn molecules are in an aggregated state (mostly as dimers).

\section{Transient Absorption Spectroscopy}

The transient absorption (TA) spectrum of a dilute solution of TPn $(0.39 \mathrm{mM})$ is displayed in Figure 4a. On the sub-nanosecond time-scale there are cooling dynamics (few ps) and anisotropy decay (137 ps). The long-lived signal is the transient spectrum of the $S_{1}$ state of TPn. The decayassociated spectra are plotted in Figure 4b (see SI for details of TA data analysis). Its evident 

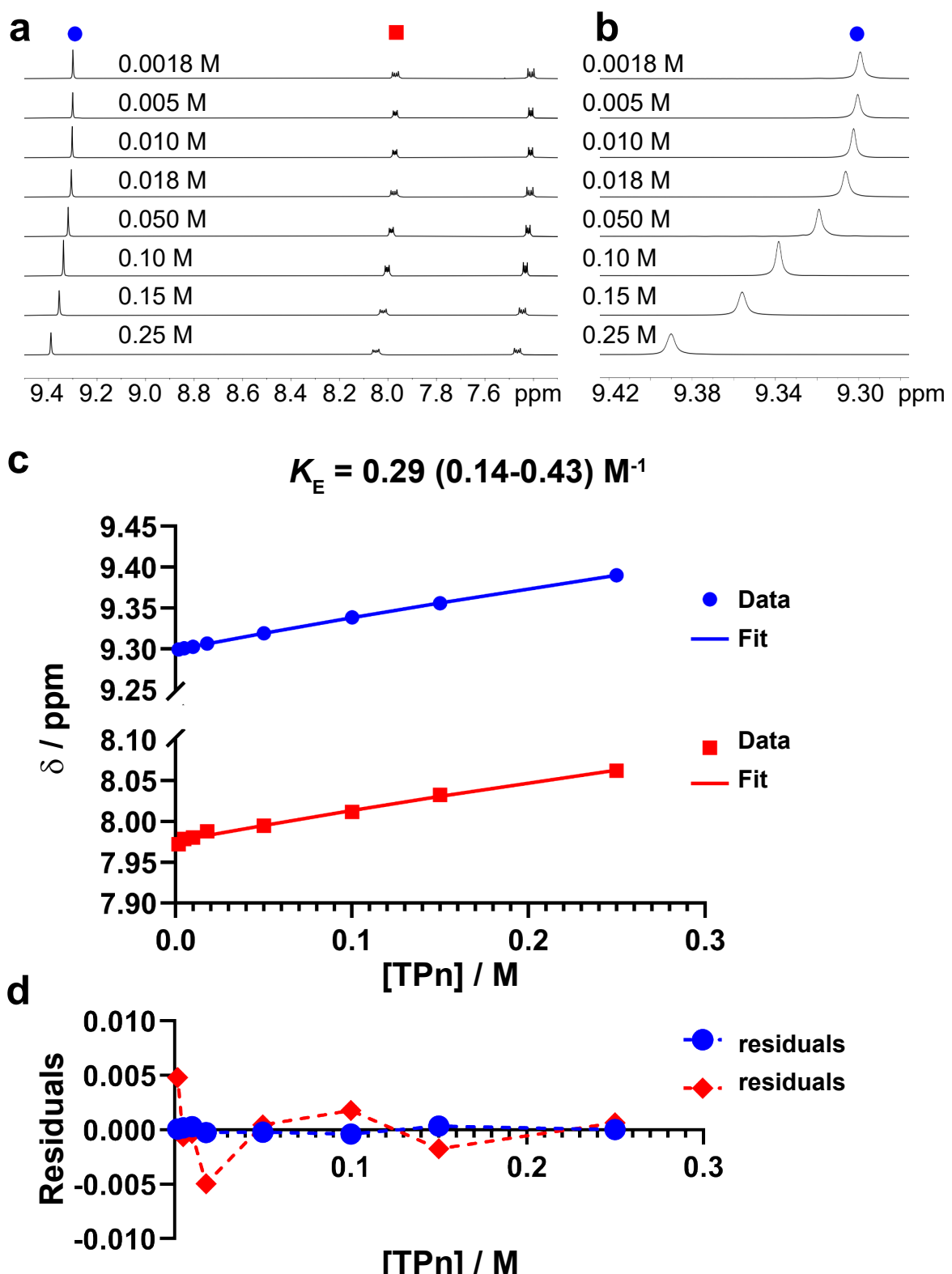

Figure 3: The aggregation of TPn. The ${ }^{1} \mathrm{H}$ NMR $\left(400 \mathrm{MHz}, \mathrm{CDCl}_{3}\right)$ spectra for a. the entire aromatic region and $\mathrm{b}$. The singlet for the 5,7,12,14-protons on TPn. c. The experimental data from a. and $b$. fitted to the EK aggregation model. The top (blue) and bottom (red) data series correspond to the relevant resonances labelled with blue circles and red squares, respectively in a. and $b$. These two dilution isotherms show the largest shift of the total of five isotherms used to fit the data to the EK-model using a global fit method (see SI). d. The residual plots obtained from the model fits shown in c. based on the EK-model with $\mathrm{KE}=0.29 \mathrm{M}^{-1}$. The $95 \%$ confidence interval (C.I.) on $K_{E}=0.14-0.43 \mathrm{M}^{-1}$, was obtained from a Monte Carlo simulation (see SI). ${ }^{28}$ 
a
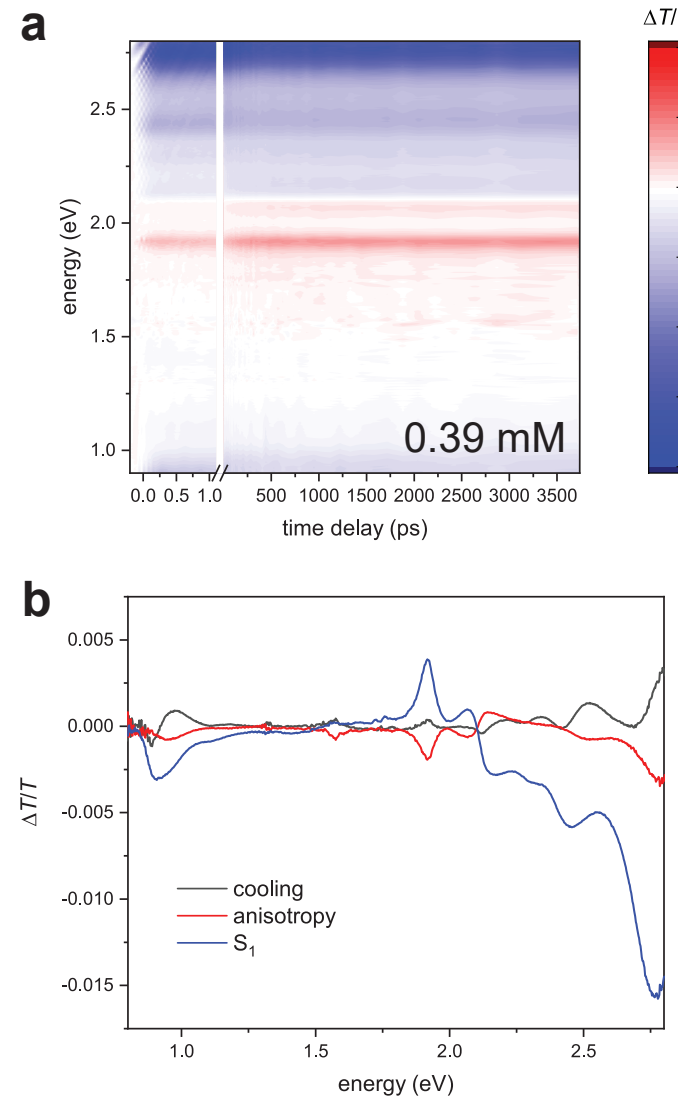
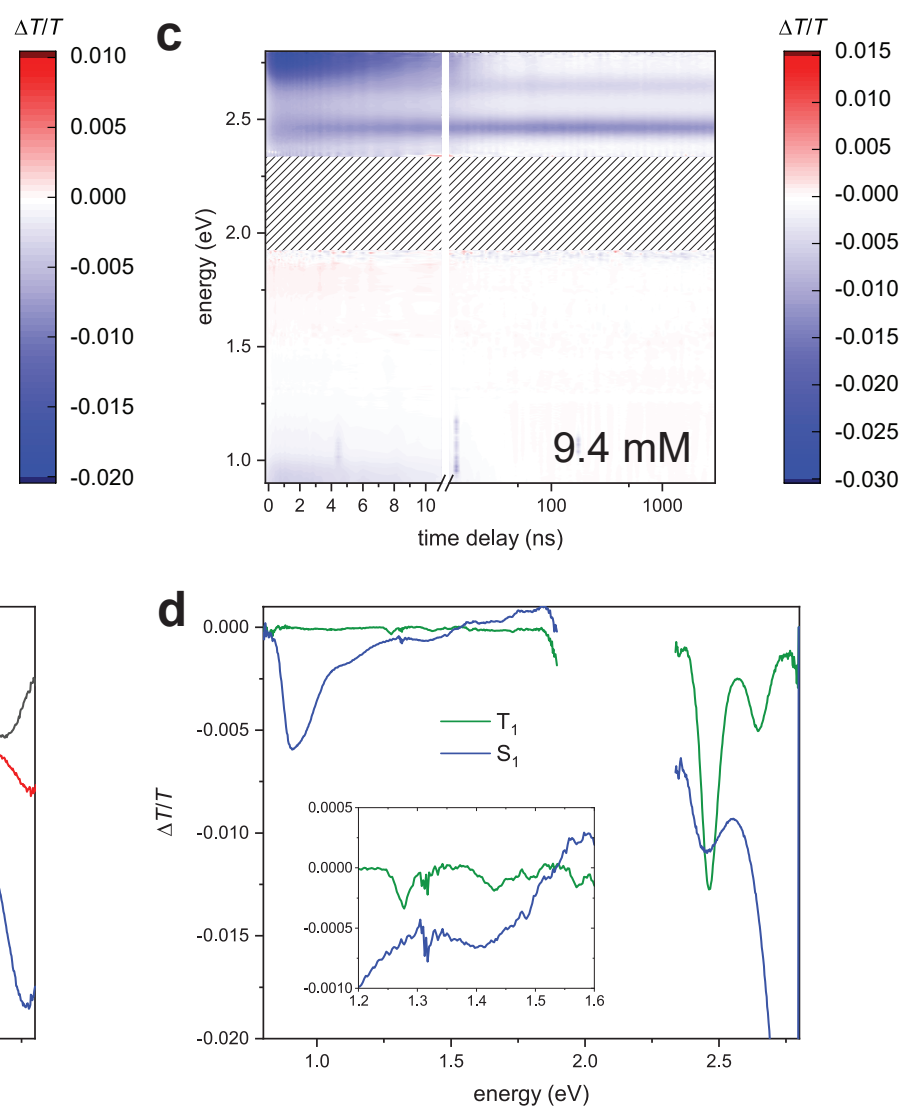

Figure 4: a. The TA spectrum of dilute TPn $(0.39 \mathrm{mM})$. b. The decay-associated spectra extracted from panel a. c. The TA spectrum of $9.4 \mathrm{mM}$ TPn. The patterned area contains no data, due to saturation of absorption. $d$. The species-associated spectra extracted from panel c. The inset shows detail of the near infrared absorptions of the $T_{1}$ state previously reported by Walker et al. ${ }^{14}$ 
ground state bleaches at 1.92 and $2.07 \mathrm{eV}$, and excited state absorptions (ESA) at $0.90 \mathrm{eV}$ and 2.17, 2.45 and $2.77 \mathrm{eV}$ accord with the spectra of Walker et al. The relative sign of the anisotropy decay compared to the transient spectrum indicates that the ESAs at 0.90, 2.45 and $2.77 \mathrm{eV}$ are polarized perpendicularly to the excitation. The bleach (as it must be) and the $2.17 \mathrm{eV}$ ESA are polarized parallel to the excitation.

At $9.4 \mathrm{mM}$ the ps TA spectrum in Figure $4 \mathrm{c}$ clearly shows the generation of a $\mu$ s-lived species which we identify as the $T_{1}$ state. The dynamics are fit to two exponentials with time constants of $\tau_{1}=10.6 \mathrm{~ns}$ and $\tau_{2}=21.5 \mu \mathrm{s}$. The extracted decay-associated spectra $\sigma_{n}$ associated with time constants $\tau_{n}$ are reconciled with sequential kinetics, revealing the species-associated spectra, $\sigma_{S_{1}}=\sigma_{1}+\sigma_{2}$ and $\sigma_{T_{1}}=\sigma_{2}$, plotted in Figure 4d. The triplet state has intense ESAs at 2.47 and $2.64 \mathrm{eV}$, and the weaker features at 1.28 and $1.43 \mathrm{eV}$, as previously identified. ${ }^{14,22}$ At $28 \mathrm{mM}$ the ps spectrum is resolved into the same components as above, but with time constants of $5.06 \mathrm{~ns}$ and $15 \mu$ s (Figure S2).

To investigate the triplet formation in detail for higher concentrations, we performed further experiments in the SpectRE Laboratory, where up to 8 ns delay can be achieved using optical delay lines, but only the near infrared region of the spectrum is detected. At $30 \mathrm{mM}$, the transient spectrum can be modelled as three decay-associated spectra. These are reconciled as a sequential decay process from (hot) $S_{1}^{*}$ to $S_{1}$ to $T_{1}$. The decay of $S_{1}$ is fit with a time constant of $6.1 \mathrm{~ns}$, in agreement with the other experiments with electronically delayed probe pulses (Figure S3).

The transient absorption spectrum of a $50 \mathrm{mM}$ solution is plotted in Figure 5a. There is an evident few picosecond decay and blue-shift of the principal ESA feature of the $S_{1}$ spectrum at $0.9-1.0 \mathrm{eV}$. This feature decays on a few nanosecond time scale, giving rise to long-lived narrow features at 1.28 and $1.43 \mathrm{eV}$ which are assigned to the triplet state. As such, one might expect the data to be well fit with two decays and an offset to account for the $T_{1}$ spectrum. The data integrated between 1.1 and $1.5 \mathrm{eV}$ is normalized in Figure $5 \mathrm{~b}$. This region focusses on the triplet features which are key to understanding singlet fission in this system. A double exponential does not resolve the initial few ps decay, and thus three exponentials are required to describe the transient. 

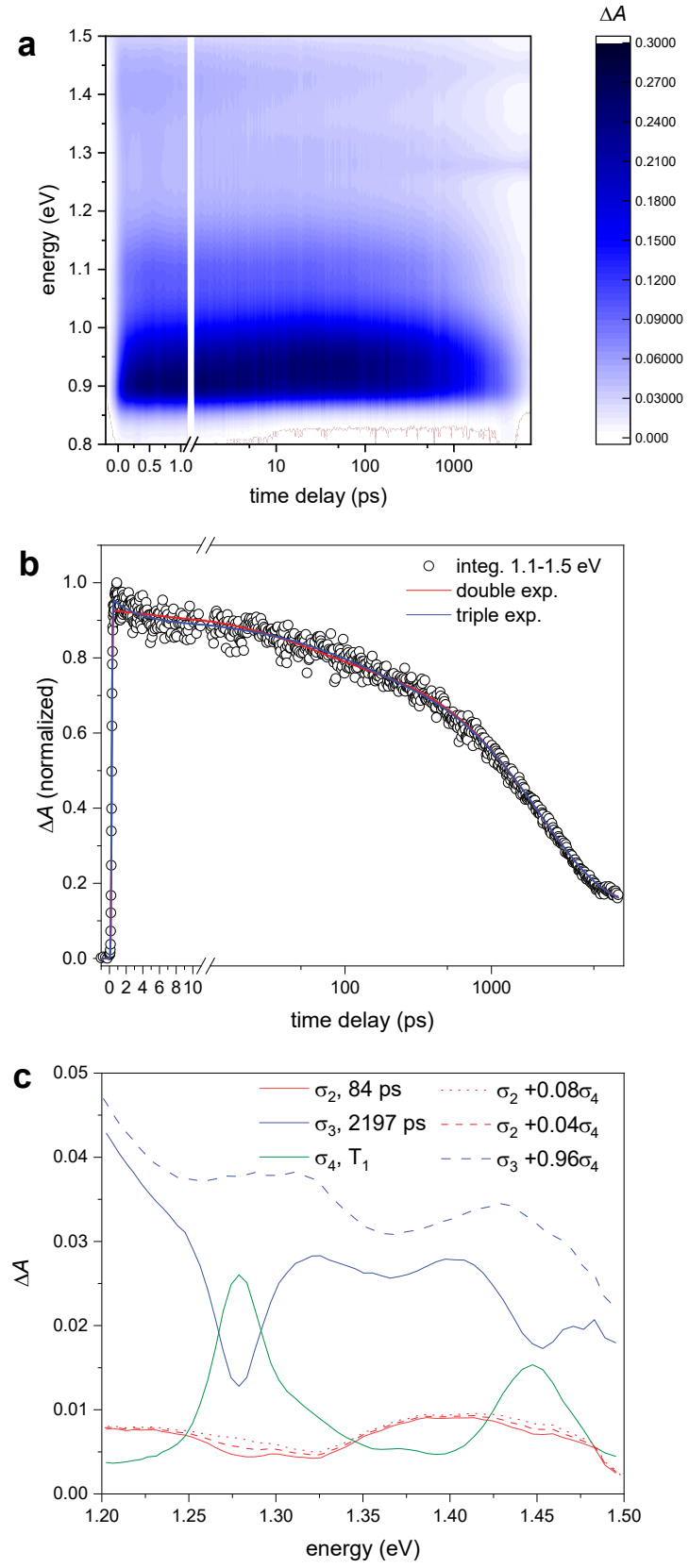

Figure 5: a. The TA spectrum of $50 \mathrm{mM}$ TPn. b. The integrated and normalized transient absorption between 1.0 and $1.5 \mathrm{eV}$ fit to a double and triple exponential decay. c. The decay-associated and species associated spectra using the time constants extracted from panel $\mathrm{b}$. 
These have time constants of 2.9(1.2), 84(15), and 2197(92) ps. The decay-associated spectra are plotted in Figure 5c.

We denote the four decay-associated spectra as $\sigma_{n}$ in order of increasing time constant $\tau_{n}$ $\left(\tau_{4}=\infty\right)$. As for other experiments, we attribute $\sigma_{1}$ to thermal equilibration of the photo-excited species. The $2197 \mathrm{ps}$ decay, $\sigma_{3}$, clearly shows a negative-going imprint of the $T_{1}$ spectrum $\left(\sigma_{4}\right)$, and is thus ascribed to singlet-fission and radiative decay of $S_{1}$ molecules. But, the magnitude of the negative going peaks may not equal those of $\sigma_{4}$. Indeed, $\sigma_{2}$ appears to have weak negativegoing triplet features. These are removed by adding $0.08 \sigma_{4}$ to $\sigma_{2}$. Similarly, the remainder of the triplet spectrum can be added to $\sigma_{3}$ to yield the spectrum of the free singlet, $S_{1}$. Species associated spectra are plotted as dotted lines in Figure 5c.

These species associated spectra would be consistent with $8 \%$ of the triplet spectrum arising on a time scale shorter than diffusion can account for. This is therefore attributed to aggregation. The NMR data indicates that an upper limit of about $4 \%$ of chromophores aggregate at $50 \mathrm{mM}$. The spectrum of $\sigma_{2}+0.04 \sigma_{4}$ is plotted in Figure 5c. It is not very different from $\sigma_{2}+0.08 \sigma_{4}$. While the level of aggregation is small, the TA and NMR results are consistent, and in agreement with Grieco et al., ${ }^{20}$ that aggregation plays a role in TIPS-pentacene solution exciton dynamics, in contrast to the study of Walker et al. that concluded that there was no evidence for aggregation on the basis of diffusion NMR experiments. The chemical shifts observed in the present experiments conclusively show aggregation is present. But, the principal mechanism for singlet fission in these solutions is the diffusion-limited encounter of an excited chromophore with ground state TPn molecules. The TCSPC results show that the lifetime of the fluorescence is shortened as a function of concentration. This dynamic quenching contrasts with the static quenching proposed by Grieco et al., who insist that pre-aggregated chromophores are responsible for singlet fission. In this case, one would observe two lifetimes, which we only start to see at the concentrations where aggregation is in evidence. 


\section{Conclusions}

We have revisited the mechanism of the singlet fission in concentrated TPn solutions. Total-internal reflection excitation showed that the emission spectrum is unchanged over a range up to $28 \mathrm{mM}$, and as such there are no observable, emissive excimers present. This contrasts strongly with the findings of Walker et al. whose emission spectra suffered severe reabsorption, ${ }^{14}$ but accords with results obtained in toluene by Schaberle et al. ${ }^{22}$

NMR experiments confirmed that aggregation occurs in TPn solutions. At the highest concentrations employed spectroscopically, only a few percent of chromophores are present as dimers, and there are hints of this in the observed transient absorption. The interactions between the chromophores, even without extensive aggregation, influence the structure of the solution, which results in a positive curve for $S_{1}$ state decay rate as a function of concentration.

TCSPC experiments showed that, at concentrations up to about $10 \mathrm{mM}$, the second order rate constant for the self-quenching of TPn is $4 \times 10^{9} \mathrm{M}^{-1} \mathrm{~s}^{-1}$, consistent with plotted data by Walker et al., ${ }^{14}$ but not with their reported rate constants, nor the finding that $50 \%$ singlet fission yield is obtained at $7.9 \mathrm{mM}$. We showed that at higher concentrations, the apparent second-order constant increases, which we rationalized in terms of the enhanced probability of finding neighbouring TPn molecules as the density increases, modelled using a simple hard-sphere approach. ${ }^{23} \mathrm{~A}$ more realistic potential would be expected to reduce the interaction distance, which was fitted here to be $2 \mathrm{~nm}$. The confirmation of dynamic quenching contrasts with the findings of Grieco et al. who reported that singlet fission does not occur by diffusive encounters. ${ }^{20}$

\section{Supporting Information}

The Supporting Information is available free of charge at https://pubs.acs.org/doi/10.1021/jacs.Xxxxx.

Fitting of transient spectroscopic data, Steady-State Absorption, NMR Dilution Studies and Data Analysis, Additional Transient Absorption Data. 


\section{Acknowledgments}

This work was supported by the Australian Research Council (Centre of Excellence in Exciton Science CE170100026). This program has been supported by the Australian Government through the Australian Renewable Energy Agency (ARENA). Responsibility for the views, information or advice expressed herein is not accepted by the Australian Government. M.D. acknowledges CAAS grant no. CZ.02.1.01/0.0/0.0/16_019/0000778.

\section{References}

(1) Smith, M. B.; Michl, J. Recent Advances in Singlet Fission. Annu. Rev. Phys. Chem. 2013, 64, 361-386.

(2) Smith, M. B.; Michl, J. Singlet Fission. Chem. Rev. 2010, 110, 6891-6936.

(3) Einzinger, M.; Wu, T.; Kompalla, J. F.; Smith, H. L.; Perkinson, C. F.; Nienhaus, L.; Wieghold, S.; Congreve, D. N.; Kahn, A.; Bawendi, M. G.; Baldo, M. A. Sensitization of silicon by singlet exciton fission in tetracene. Nature 2019, 571, 90-94.

(4) MacQueen, R. W.; Liebhaber, M.; Niederhausen, J.; Mews, M.; Gersmann, C.; Jäckle, S.; Jäger, K.; Tayebjee, M. J. Y.; Schmidt, T. W.; Rech, B.; Lips, K. Crystalline silicon solar cells with tetracene interlayers: the path to silicon-singlet fission heterojunction devices. Mater. Horiz. 2018, 5, 1065-1075.

(5) Tayebjee, M. J. Y.; McCamey, D. R.; Schmidt, T. W. Beyond Shockley-Queisser: Molecular Approaches to High-Efficiency Photovoltaics. J. Phys. Chem. Lett. 2015, 6, 2367-2378.

(6) Rao, A.; Friend, R. H. Harnessing singlet exciton fission to break the Shockley-Queisser limit. Nature Rev. Mater. 2017, 2, 17063.

(7) Tayebjee, M. J. Y.; Gray-Weale, A. A.; Schmidt, T. W. Thermodynamic Limit of Exciton Fission Solar Cell Efficiency. J. Phys. Chem. Lett. 2012, 3, 2749-2754. 
(8) Piland, G. B.; Bardeen, C. J. How Morphology Affects Singlet Fission in Crystalline Tetracene. J. Phys. Chem. Lett. 2015, 6, 1841-1846.

(9) Dover, C. B.; Gallaher, J. K.; Frazer, L.; Tapping, P. C.; Petty, A. J.; Crossley, M. J.; Anthony, J. E.; Kee, T. W.; Schmidt, T. W. Endothermic singlet fission is hindered by excimer formation. Nature Chemistry 2018, 10, 305-310.

(10) Rao, A.; Wilson, M. W. B.; Albert-Seifried, S.; Di Pietro, R.; Friend, R. H. Photophysics of pentacene thin films: The role of exciton fission and heating effects. Phys. Rev. B 2011, 84, 195411.

(11) Tayebjee, M. J. Y.; Schwarz, K. N.; MacQueen, R. W.; Dvořák, M.; Lam, A. W. C.; Ghiggino, K. P.; McCamey, D. R.; Schmidt, T. W.; Conibeer, G. J. Morphological Evolution and Singlet Fission in Aqueous Suspensions of TIPS-Pentacene Nanoparticles. J. Phys. Chem. C 2016, 120, 157-165.

(12) Wilson, M. W. B.; Rao, A.; Ehrler, B.; Friend, R. H. Singlet Exciton Fission in Polycrystalline Pentacene: From Photophysics toward Devices. Acc. Chem. Res. 2013, 46, 1330-1338.

(13) Pensack, R. D.; Tilley, A. J.; Parkin, S. R.; Lee, T. S.; Payne, M. M.; Gao, D.; Jahnke, A. A.; Oblinsky, D. G.; Li, P.-F.; Anthony, J. E.; Seferos, D. S.; Scholes, G. D. Exciton Delocalization Drives Rapid Singlet Fission in Nanoparticles of Acene Derivatives. J. Am. Chem. Soc. 2015, 137, 6790-6803.

(14) Walker, B. J.; Musser, A. J.; Beljonne, D.; Friend, R. H. Singlet exciton fission in solution. Nature Chemistry 2013, 5, 1019-1024.

(15) Stern, H. L.; Musser, A. J.; Gelinas, S.; Parkinson, P.; Herz, L. M.; Bruzek, M. J.; Anthony, J.; Friend, R. H.; Walker, B. J. Identification of a triplet pair intermediate in singlet exciton fission in solution. Proc. Natl. Acad. Sci. 2015, 112, 7656-7661. 
(16) Schrauben, J. N.; Ryerson, J. L.; Michl, J.; Johnson, J. C. Mechanism of Singlet Fission in Thin Films of 1,3-Diphenylisobenzofuran. J. Am. Chem. Soc. 2014, 136, 7363-7373.

(17) Mauck, C. M.; Hartnett, P. E.; Margulies, E. A.; Ma, L.; Miller, C. E.; Schatz, G. C.; Marks, T. J.; Wasielewski, M. R. Singlet Fission via an Excimer-Like Intermediate in 3,6Bis(thiophen-2-yl)diketopyrrolopyrrole Derivatives. J. Am. Chem. Soc. 2016, 138, 1174911761 .

(18) Korovina, N. V.; Das, S.; Nett, Z.; Feng, X.; Joy, J.; Haiges, R.; Krylov, A. I.; Bradforth, S. E.; Thompson, M. E. Singlet Fission in a Covalently Linked Cofacial Alkynyltetracene Dimer. J. Am. Chem. Soc. 2016, 138, 617-627.

(19) Bossanyi, D. G.; Matthiesen, M.; Wang, S.; Smith, J. A.; Kilbride, R. C.; Shipp, J. D.; Chekulaev, D.; Holland, E.; Anthony, J. E.; Zaumseil, J.; Musser, A. J.; Clark, J. Emissive spin-0 triplet-pairs are a direct product of triplet-triplet annihilation in pentacene single crystals and anthradithiophene films. Nature Chem. 2020, 13, 163-171.

(20) Grieco, C.; Doucette, G. S.; Munson, K. T.; Swartzfager, J. R.; Munro, J. M.; Anthony, J. E.; Dabo, I.; Asbury, J. B. Vibrational probe of the origin of singlet exciton fission in TIPSpentacene solutions. J. Chem. Phys. 2019, 151, 154701.

(21) Gholizadeh, E. M.; Prasad, S. K. K.; Teh, Z. L.; Ishwara, T.; Norman, S.; Petty II, A. J.; Cole, J. H.; Cheong, S.; Tilley, R. D.; Anthony, J. E.; Huang, S.; Schmidt, T. W. Photochemical upconversion of near-infrared light from below the silicon bandgap. Nature Photonics 2020, 14, 585-590.

(22) Schaberle, F. A.; Serpa, C.; Arnaut, L. G.; Ward, A. D.; Karlsson, J. K. G.; Atahan, A.; Harriman, A. The Photophysical Properties of Triisopropylsilyl-ethynylpentacene-A Molecule with an Unusually Large Singlet-Triplet Energy Gap-In Solution and Solid Phases. Chemistry 2020, 2, 545-564. 
(23) Keizer, J. Nonlinear fluorescence quenching and the origin of positive curvature in SternVolmer plots. J. Am. Chem. Soc. 1983, 105, 1494-1498.

(24) Keizer, J. Diffusion Effects on Rapid Bimolecular Chemical Reactions. Chem. Rev. 1987, 87, $167-180$.

(25) Throop, G. J.; Bearman, R. J. Numerical Solutions of the Percus-Yevick Equation for the Hard-Sphere Potential. The Journal of Chemical Physics 1965, 42, 2408-2411.

(26) Lebowitz, J. L. Exact Solution of Generalized Percus-Yevick Equation for a Mixture of Hard Spheres. Phys. Rev. 1964, 133, A895-A899.

(27) Monroe, B. M. photochemical estimation of oxygen solubility. Photochem. Photobiol. 35, $863-865$.

(28) Hibbert, D. B.; Thordarson, P. The death of the Job plot, transparency, open science and online tools, uncertainty estimation methods and other developments in supramolecular chemistry data analysis. Chem. Commun. 2016, 52, 12792-12805.

(29) Martin, R. B. Comparisons of Indefinite Self-Association Models. Chem. Soc. Rev. 1996, 8, 3043-3064.

(30) http://supramolecular.org.

(31) Thordarson, P. In Supramolecular Chemistry: From Molecules to Nanomaterials; Steed, J. W., Gale, P. A., Eds.; John Wiley \& Sons, Chichester, UK, 2012; Vol. 2; Chapter Binding Constants and their Measurement, pp 239-274.

(32) Schwarz, G. Estimating the Dimension of a Model. The Annals of Statistics 1978, 6, 461-464. 


\section{Graphical TOC Entry}

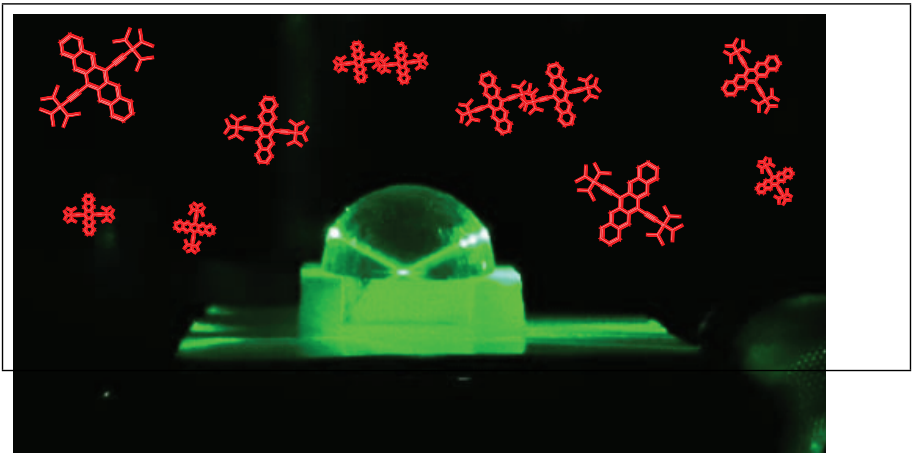




\title{
Supporting Information:
}

\section{Singlet fission in concentrated TIPS-pentacene solutions: The role of excimers and aggregates}

\author{
Miroslav Dvořák, ${ }^{\dagger, \ddagger}$ Shyamal K. K. Prasad, ${ }^{\dagger}$ Cameron B. Dover, ${ }^{\dagger}$ Chelsea R. Forest, ${ }^{\circledR}$ Akasha \\ Kaleem, ${ }^{\S}$ Rowan W. MacQueen, ${ }^{\prime}$ Anthony J. Petty II, ${ }^{\perp}$ Roslyn Forecast, ${ }^{\#}$ John E. Anthony, ${ }^{\perp}$ \\ Murad J. Y. Tayebjee, ${ }^{\S}$ Asaph Widmer-Cooper, ${ }^{@}$ Pall Thordarson, ${ }^{\uparrow}$ and Timothy W. Schmidt*,† \\ $\dagger$ †ARC Centre of Excellence in Exciton Science, School of Chemistry, UNSW Sydney, NSW 2052, Australia \\ $\ddagger$ Department of Physical Electronics, Faculty of Nuclear Sciences and Physical Engineering, Czech Technical \\ University in Prague, V Holešovičkách 2, 18000 Prague 8, Czechia \\ TAustralian Centre for Nanomedicine and The ARC Centre of Excellence in Convergent Bio-Nano Science and \\ Technology, School of Chemistry, UNSW Sydney, NSW 2052, Australia \\ $\S$ School of Photovoltaic and Renewable Energy Engineering, UNSW Sydney, NSW 2052, Australia \\ ||Department of Spins in Energy Conversion and Quantum Information Science, Helmholtz-Zentrum Berlin für \\ Materialen und Energie GmbH, Berlin, Germany \\ $\perp$ Department of Chemistry, University of Kentucky, Lexington, Kentucky 40506, United States \\ \#ARC Centre of Excellence in Exciton Science, School of Science, RMIT University, Melbourne, VIC, 3001,
}

Australia

@ARC Centre of Excellence in Exciton Science, School of Chemistry, The University of Sydney, NSW 2006, Australia

E-mail: timothy.schmidt@unsw.edu.au 


\section{Fitting of Transient Spectroscopic Data}

The transient absorption experiments generate signals which are functions of time delay, $t$, and photon energy, $E$ : $\Sigma(E, t)$. We assume that the signal ( $\Delta A$ or $\Delta T / T)$ at any photon energy can be fit to multiple exponential decays.

The instrument response function is modelled using the error function,

$$
F(E, t)=0.5\left(1+\operatorname{erf}\left(\left(t-t_{0}(E)\right) / \tau_{p}\right)\right)
$$

and any chirp is fit to a quadratic function in energy (or wavelength),

$$
t_{0}=c+b E+a E^{2}
$$

As such, the fitting function is

$$
\Sigma(E, t)=F(E, t) \sum_{i} \sigma_{i}(E) \exp \left(-\left(t-t_{0}(E)\right) / \tau_{i}\right)
$$

\section{Decay-Associated Spectra}

The $\sigma_{i}(E)$ are decay-associated spectra, which decay with time constant $\tau_{i}$, and do not necessarily

correspond to particular species. The correspondence with species-associated spectra must be obtained by application of a kinetic model.

The relationship between $\Delta A$ and $\Delta T / T$ is obtained by

$$
\begin{aligned}
A & =-\frac{\log (T)}{\log (10)} \\
\Delta A & =-\frac{1}{\log (10)} \frac{\Delta T}{T}
\end{aligned}
$$




\section{Species-Associated Spectra}

\section{One Time Constant}

Where there is only one time constant, $\tau_{i}$, the decay-associated spectrum (DAS) is also the speciesassociated spectrum (SAS), unless there are two species that decay with identical time constants.

\section{Two Time Constants}

Where there are two time constants, there should be at least two species. In the case of two species, these may both be formed by the laser pulse, or one may be formed by the other. In the former case, the SAS are the DAS. In the latter case, one must consider the time dependence of each species. 


\section{Steady-State Absorption}

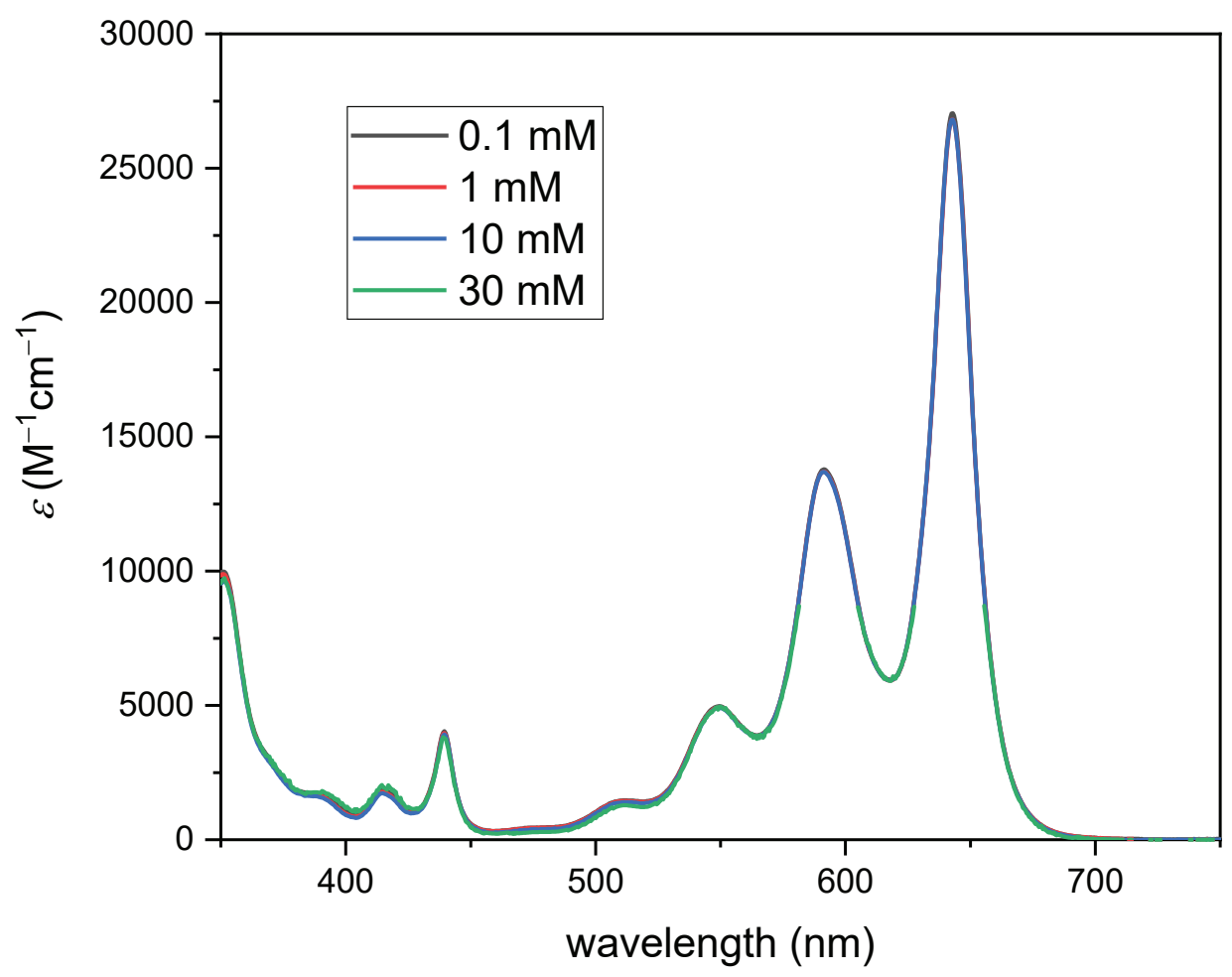

Figure S1: The absorption spectrum of TIPS-pentacene at four concentrations. All measurements but one were performed in $0.1 \mathrm{~mm}$ cuvette. The $0.1 \mathrm{mM}$ measurement was performed in a $10 \mathrm{~mm}$ cuvette. The $30 \mathrm{mM}$ spectrum was cut off at OD 2.2. The max epsilon is $27000 \mathrm{M}^{-1} \mathrm{~cm}^{-1}$. 


\section{NMR Dilution Studies and Data Analysis}

The data were fit to two different aggregation models, the EK-model (isodesmic or non-cooperative aggregating model) ${ }^{\mathrm{S} 1}$ and the coEK (cooperative aggregation model). ${ }^{\mathrm{S} 1, \mathrm{~S} 2}$ The results from the two different models used to fit the data can be accessed via the www.supramolecular.org ${ }^{\mathrm{S} 3}$ database through the unique URL below (copy-paste into a web-browser):

EK-model (isodesmic or non-cooperative aggregating model):

http://app.supramolecular.org/bindfit/view/68c05efe-alc8-44a8-aea3-86b664b4ba5a coEK (cooperative aggregation model)

http://app.supramolecular.org/bindfit/view/a0815aeb-1ee2-47c2-a78a-88b186c37abd

Table S1: Comparison of The Models

\begin{tabular}{|c|c|}
\hline 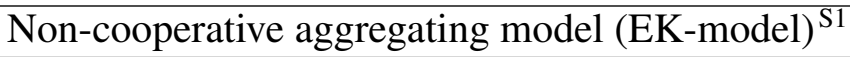 & (1 parameter) \\
\hline$K_{E}$ & $0.29 \mathrm{M}^{-1}$ \\
\hline $\mathrm{BIC}^{\mathrm{S} 4}$ & -391 \\
\hline Aggregate $\%$ at $0.028 \mathrm{M}$ & $1.6 \%$ \\
\hline Cooperative aggregation model (coEK model) ${ }^{\mathrm{S} 1, \mathrm{~S} 2}$ & (2 parameters) \\
\hline$K_{E}$ & $0.15 \mathrm{M}^{-1}$ \\
\hline$\rho\left(K_{2}=K\right)$ & 1.5 \\
\hline $\mathrm{BIC}^{\mathrm{S} 4}$ & -387 \\
\hline Aggregate $\%$ at $0.028 \mathrm{M}$ & $1.2 \%$ \\
\hline
\end{tabular}

Non-cooperative aggregating model; EK-model, fits better according to BIC (Bayesian Information Criteria) $^{\mathrm{S} 4}$ than the more complex coEK model as the BIC for the EK-model is more negative (lower).

\section{Uncertainty estimation by Monte Carlo simulation}

Based on our previous work, ${ }^{\mathrm{S} 5}$ to estimate the uncertainty, a Monte Carlo simulation (20000 runs) was based on random sampling from distributions of the input concentration data for TIPSpentacene, and the measured NMR data (all 5 isotherms $\times 8$ different concentrations $=40$ data points). The relative standard deviations of the distributions were $2 \%$ for the TIPS-pentacene concentration and $\pm 0.001 \mathrm{ppm}$ for the measured NMR data. The resulting $20000 K_{E}$ values were 
ranked and the bottom $2.5 \%$ and $97.5 \%$ extracted to obtain the lower and upper 95\% confidence bounds as:

95\% confidence interval according to Monte Carlo simulation: $K_{E}=0.14^{\backsim} 0.46 \mathrm{M}^{-1}$.

The mean and median were $0.29 \mathrm{M}^{-1}$ in line with the "best fit".

The raw data, the Matlab files used to run the Monte Carlo simulation and the large output file are all accessible at github:

https://github.com/pallithordarson/NMRdilution-TIPS-pentacene/

To rerun the Monte Carlo simulation, upload the files from Github, then load the data in the Matlab workspace with $->$ load nmrdilutiondata on the command line, followed by -> MonteCarloNMRdilution to run the actual Monte Carlo simulation. 


\section{Transient Absorption}

a
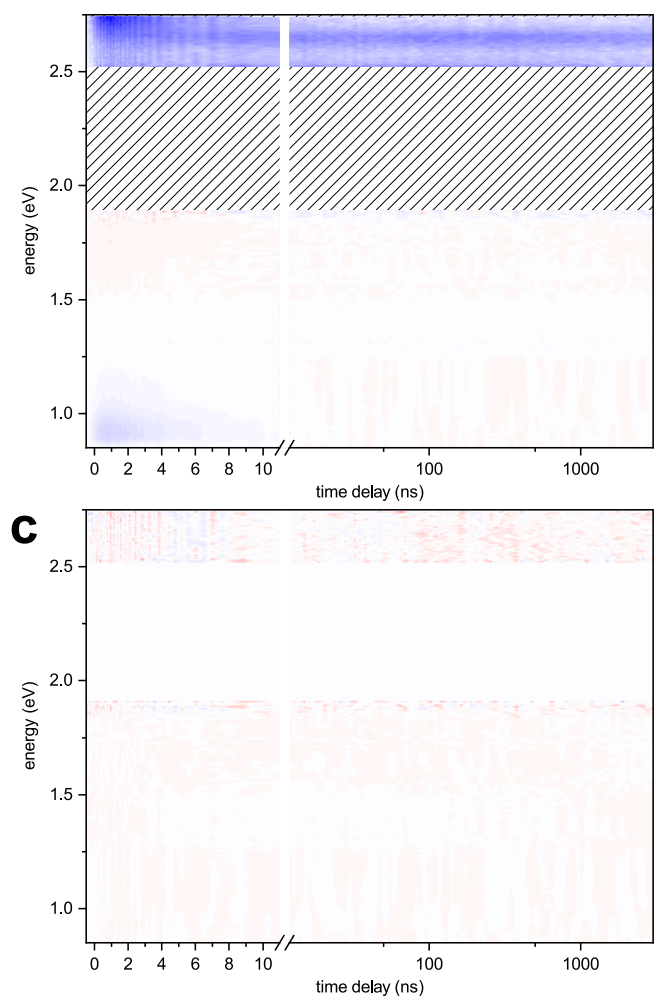

b
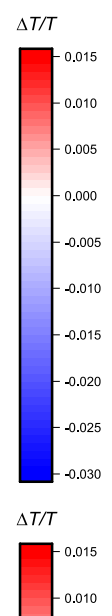

$\underbrace{0.015}_{-0.005}$

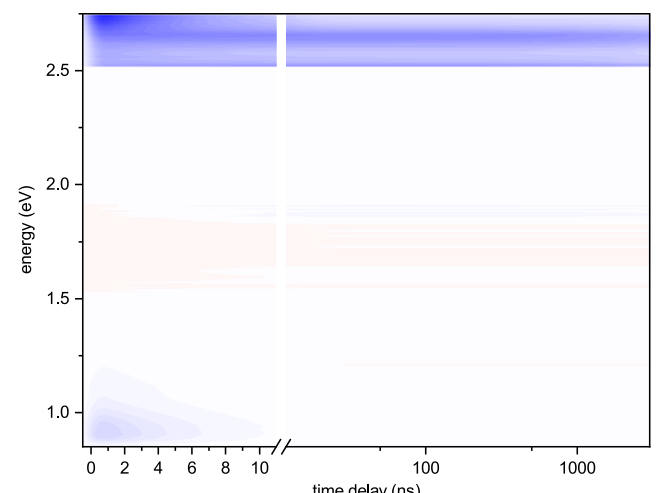

$\Delta T / T$

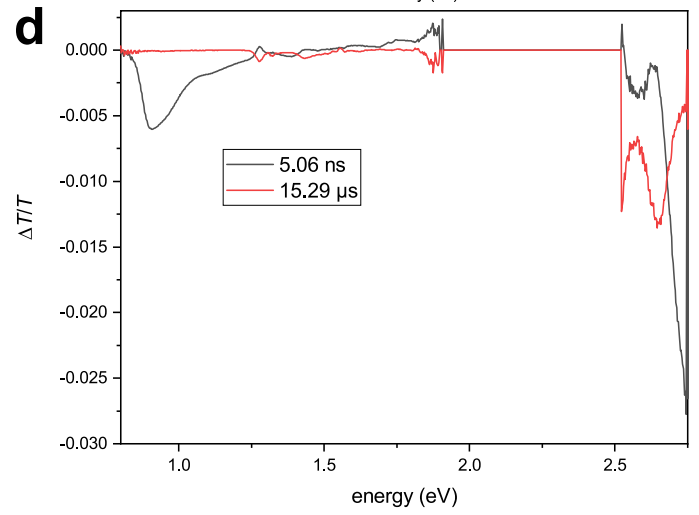

Figure S2: a. Picosecond transient absorption spectra of $28 \mathrm{mM}$ TPn obtained in the Chemistry laboratory. b. Synthetic spectrum fit using two time constants. c. Residuals of fit. d. Decayassociated spectra. 

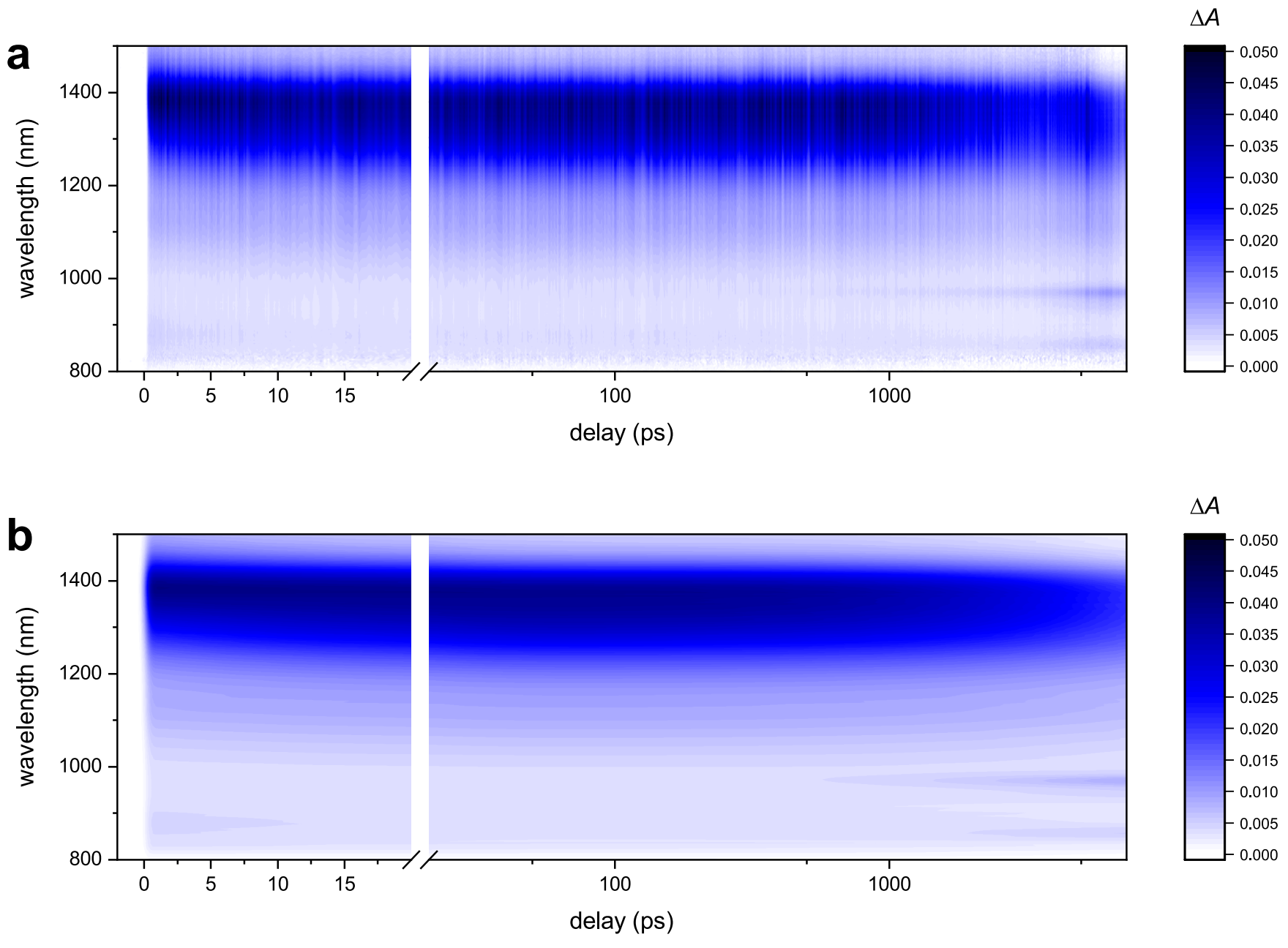

Figure S3: a. Femtosecond transient absorption spectra of $30 \mathrm{mM}$ TPn obtained in the Photovoltaic laboratory. b. Synthetic spectrum fit using three time constants: $14 \mathrm{ps}, 6.1 \mathrm{~ns}$ and $3.4 \mu \mathrm{s}$ (much longer than the range scanned). 


\section{References}

(S1) Martin, R. B. Comparisons of Indefinite Self-Association Models. Chem. Soc. Rev. 1996, 8, 3043-3064.

(S2) Thordarson, P. In Supramolecular Chemistry: From Molecules to Nanomaterials; Steed, J. W., Gale, P. A., Eds.; John Wiley \& Sons, Chichester, UK, 2012; Vol. 2; Chapter Binding Constants and their Measurement, pp 239-274.

(S3) http://supramolecular.org.

(S4) Schwarz, G. Estimating the Dimension of a Model. The Annals of Statistics 1978, 6, 461-464.

(S5) Hibbert, D. B.; Thordarson, P. The death of the Job plot, transparency, open science and online tools, uncertainty estimation methods and other developments in supramolecular chemistry data analysis. Chem. Commun. 2016, 52, 12792-12805. 
